\title{
SIMPLE EXAMPLES OF NONREALIZABLE CR HYPERSURFACES
}

\author{
HOWARD JACOBOWITZ
}

\begin{abstract}
A new proof is provided of a nonrealizability result due to Hill, Penrose, and Sparling. This result is then generalized to higher dimensions: Each $\bar{\partial}_{b}$-cohomology class in $H^{0,1}(M)$ can be used to define a nonrealizable CR structure on $M \times \mathbf{R}^{2}$.
\end{abstract}

Consider an example due to Hill, Penrose, and Sparling $[\mathbf{P}]$ as formulated by Eastwood [E]. Let $M$ be a three-dimensional hypersurface in $\mathbf{C}^{2}$ with Lewy operator $L$. Define a $\mathrm{CR}$ structure on $M \times \mathbf{C}$ by taking

$$
L_{1}=L+g \zeta \partial / \partial \zeta, \quad L_{2}=\partial / \partial \bar{\zeta}
$$

where $g$ is a function on $M$ and $\zeta$ is the coordinate for $\mathbf{C}$. It is known that when $L f=g$ has no solution, then this structure cannot be realized as a hypersurface in $\mathbf{C}^{3}$. The proof outlined in $[\mathbf{P}]$ uses the extension of holomorphic vector bundles across boundaries in the base. A simple proof using a Taylor series expansion was given in [E]. Here we give another simple proof; this one uses the canonical bundle $K$ of a CR manifold. This bundle has also been useful in other contexts $[\mathbf{F}, \mathbf{J}]$. If $M^{2 n+1}$ is a CR manifold of hypersurface type and if $\theta_{1}, \theta_{2}, \ldots, \theta_{n+1}$ are independent forms each of which annihilates every Lewy vector field, then $K=$ $\left\{\lambda \theta_{1} \wedge \cdots \wedge \theta_{n+1}, \lambda \in \mathbf{C}^{*}\right\}$. Note that $K$ does not depend on the particular choices of $\theta_{j}$.

So now let $\theta_{1}=d z_{1}$ and $\theta_{2}=d z_{2}$ where $\left(z_{1}, z_{2}\right)$ are the coordinates on $\mathbf{C}^{2}$ restricted to $M$. We may assume $d z_{1} d \bar{z}_{1} d z_{2} \neq 0$ and also that $L z_{1}=0, L z_{2}=0$, $L \bar{z}_{1}=1$. Let $\theta_{3}=d \zeta-g \zeta \bar{\theta}_{1}$ and $\Omega=\theta_{1} \theta_{2} \theta_{3}$. Thus $\Omega$ is a section of the canonical bundle of $M \times \mathbf{C}$. If $M \times \mathbf{C}$ can be realized by a hypersurface in $\mathbf{C}^{3}$ then, using $\left(w_{1}, w_{2}, w_{3}\right)$ as coordinates on $\mathbf{C}^{3}, d w_{1} d w_{2} d w_{3}$ is also a section of this bundle and so is a multiple of $\Omega$. Thus $d(f \Omega)=0$ for some nonzero function $f$. But

$$
d(f \Omega)=\left(L f+f g+f_{\zeta} g \zeta\right) \bar{\theta}_{1} \Omega+f_{\bar{\zeta}} \bar{\theta}_{3} \Omega .
$$

In particular, we may set $\zeta=0$ to obtain $L(-\ln f)=g$. Thus if $M \times \mathbf{C}$ is realizable then $L f=g$ is solvable. Conversely, if $L f=g$ then $\left(z_{1}, z_{2}, \zeta e^{-f}\right)$ provides an embedding into $\mathbf{C}^{3}$.

It is simple to give a generalization to higher dimensions. Let $M^{2 n+1}$ be a hypersurface in $\mathbf{C}^{n+1}$. Let $g$ be a 1 -form on $M$ which is a representative of a $\bar{\partial}_{b}$-cohomology class $[g]$ in $H^{0,1}(M)$. Let $\left(z_{1}, \ldots, z_{n+1}\right)$ be coordinates on $\mathbf{C}^{n+1}$

Received by the editors October 15, 1985.

1980 Mathematics Subject Classification (1985 Revision). Primary 32F99, 35F05.

This work was partially supported by NSF grant DMS 84-02631 and was carried out while the author was a Member at The Institute for Advanced Study.

(C) 1986 American Mathematical Society $0002-9939 / 86 \$ 1.00+\$ .25$ per page 
and $\varsigma$ a coordinate on $\mathbf{C}$. Note that $d z_{1} \cdots d z_{n+1}$ is nonzero when restricted to $M$. Define a $\mathrm{CR}$ structure on $M \times \mathbf{C}$ by setting

$$
\theta_{j}=d z_{j}, \quad j=1, \ldots, n+1, \quad \text { and } \quad \theta_{n+2}=d \varsigma-\varsigma g .
$$

THEOREM. This $C R$ structure is integrable and depends only on $[g]$. It is nonrealizable precisely when $[g] \neq 0$.

REMARK. If $M$ is nondegenerate hypersurface of "signature" $(1, n-1)$ then $H^{0,1}$ is not zero and so there exists a degenerate nonrealizable CR structure of signature $(1, n-1,0)$.

PROOF. Since $g$ is taken to represent a cohomology class we have that $d g=$ $\sum_{1}^{n+1} g_{j} \wedge \theta_{j}$. Thus $d \theta_{n+2}=-d \varsigma \wedge g-\zeta d g=-\theta_{n+2} \wedge g-\varsigma\left(g_{j} \wedge \theta_{j}\right)$. So $d \theta_{j} \in$ $\left\{\theta_{1}, \ldots, \theta_{n+2}\right\}$ and our structure is integrable. Let $g$ and $h$ represent the same class in $H^{0,1}$. So $g=h+\sum_{1}^{n+1} \alpha_{j} \theta_{j}+d f$ for some functions on $M$. Let $\omega=d \eta-\eta h$ for $\eta$ a complex variable. We claim that $S=\left\{\theta_{1}, \ldots \theta_{n+2}\right\}$ and $S^{\prime}=\left\{\theta_{1} \cdots \theta_{n+1}, \omega\right\}$ define the same $\mathrm{CR}$ structure. By this we mean there is a map $\Phi: M \times \mathbf{C} \rightarrow M \times \mathbf{C}$ such that $\Phi_{*}\left(\theta_{j}\right) \in$ linear span $S^{\prime}$. Let $\Phi(z, \eta)=\left(z, \eta e^{f}\right)$. So $\Phi_{*} \theta_{j}=\theta_{j}, j=1, \ldots, n+1$, and

$$
\Phi_{*}\left(\theta_{n+2}\right)=d\left(\eta e^{f}\right)-\eta e^{f} g=e^{f} \omega \bmod \left\{\theta_{1}, \ldots, \theta_{n+1}\right\} .
$$

Thus $\Phi_{*}\left(\theta_{n+2}\right) \in S^{\prime}$. In particular, if $[g]=0$ then $g$ and 0 define the same CR structure. This structure is now the product of the CR structures $M \times \mathbf{C}$ and hence is realizable in $\mathbf{C}^{n+2}$. Thus it only remains to show that if the $\mathrm{CR}$ structure given by $S$ is realizable then $[g]=0$. Let $\Omega=\theta_{1} \cdots \theta_{n+2}$ be a section of the canonical bundle. Since $\theta_{1} \cdots \theta_{n+1} d g=0$, we have that $d \Omega=g \wedge \Omega$. But, by the same reasoning as before, if $M \times \mathbf{C}$ is realizable, then $d(f \Omega)=0$ for some function $f$. Thus

$$
\left(d^{\prime} f+f g+f_{\zeta} \zeta g+f_{\bar{\zeta}} \bar{\zeta} \bar{g}+f_{\bar{\zeta}} \bar{\theta}_{n+2}\right) \Omega=0
$$

where $d^{\prime}$ is with respect to $M$. Set $\zeta=0$ to obtain $\left(d^{\prime}(-\ln f)-g\right) \theta_{1} \cdots \theta_{n+1}=0$. This implies $[g]=0$.

The author would like to thank Claude LeBrun for bringing to his attention the particular formulation in [E] of our example (1). Our proof and generalization follow naturally from the knowledge that (1) is nonrealizable. The harder task, accomplished by Hill, Penrose, and Sparling, was to find this example.

\section{REFERENCES}

[E] M. Eastwood, The Hill-Penrose-Sparling C.R.-folds, Twistor Newsletter 18 (1984), 16.

[F] F. Farris, An intrinsic construction of the Fefferman metric (to appear).

[J] H. Jacobowitz, The canonical bundle of a CR manifold, Pacific J. Math. (to appear).

[P] R. Penrose, Physical space-time and nonrealizable CR-structures, Bull. Amer. Math. Soc. 8 (1983), 427-448. 